\title{
In situ structure of FtsZ mini-rings in Arabidopsis chloroplasts
}

\author{
Carol B. Johnson' ${ }^{1}$, Zheng Long ${ }^{2}$, Zhiping Luo ${ }^{3}$, Rahamthulla S. Shaik ${ }^{1}$, Min Woo Sung ${ }^{1}$, Stanislav Vitha ${ }^{4}$ \\ and Andreas Holzenburg ${ }^{1,4,5^{*}}$
}

\begin{abstract}
Chloroplasts are essential plant organelles that divide by binary fission through a coordinated ring-shaped division machinery located both on the outside and inside of the chloroplast. The first step in chloroplast division is the assembly of an internal division ring (Z-ring) that is composed of the key filamentous chloroplast division proteins FtsZ1 and FtsZ2. How the individual FtsZ filaments assemble into higher-order structures to form the dividing Z-ring is not well understood and the most detailed insights have so far been gleaned from prokaryotic FtsZ. Here, we present in situ data of chloroplast FtsZ making use of a smaller ring-like FtsZ assembly termed mini-rings that form under well-defined conditions. Structured illumination microscopy (SIM) permitted their mean diameter to be determined as $208 \mathrm{~nm}$ and also showed that $68 \%$ of these rings are terminally attached to linear FtsZ filaments. A correlative microscopy-compatible specimen preparation based on freeze substitution after high-pressure freezing is presented addressing the challenges such as autofluorescence and specific fluorescence attenuation. Transmission electron microscopy (TEM) and scanning TEM (STEM) imaging of thin sections exhibited ring-like densities that matched in size with the SIM data, and TEM tomography revealed insights into the molecular architecture of mini-rings demonstrating the following key features: (1) overall, a roughly bipartite split into a more ordered/curved and less ordered/curved half is readily discernible; (2) the density distribution in individual strands matches with the X-ray data, suggesting they constitute FtsZ protofilaments; (3) in the less ordered half of the ring, the protofilaments are able to assemble into higher-order structures such as double helices and supercoiled structures. Taken together, the data suggest that the state of existence of mini-rings could be described as metastable and their possible involvement in filament storage and Z-ring assembly is discussed.
\end{abstract}

Keywords: Chloroplast division, Correlative imaging, FtsZ mini-rings, Fluorescence microscopy, Transmission electron microscopy, Electron tomography, Structured illumination microscopy, High-pressure freezing, Freeze substitution

\section{Background}

Chloroplasts are essential plant organelles that arose from cyanobacterial ancestors by the process of endosymbiosis. Chloroplast division is required to maintain both the size and number of chloroplasts, which in turn affect photosynthetic performance of the plant via the control of surface-to-volume ratio with larger ratios facilitating the exchange of metabolites between the chloroplast and the cytosol. The size of starch-storing plastids

\footnotetext{
${ }^{*}$ Correspondence: holzen@tamu.edu

${ }^{4}$ Microscopy and Imaging Center, Texas A\&M University, Interdisciplinary Life Sciences Building, 2257 TAMU, College Station, TX 77843-2257, USA Full list of author information is available at the end of the article
}

(amyloplasts) affects the properties of starch granules. Controlling the latter in crops is of interest to industries concerned with the applications of starch $[1,2]$, and to this end it is critical to understand by what mechanism plastid/chloroplast size is controlled. Basically, both bacteria and chloroplasts divide by the process of binary fission. Following on from that, the division process is initiated by assembly of the cytoskeletal protein FtsZ into filaments that form a ring structure, the Z-ring, at the division site $[3,4]$. Plants and algae encode two conserved FtsZ families, FtsZ1 and FtsZ2 [5-7], which are functionally different and are both required for plastid division and plastid size control. Changes in FtsZ expression levels or assembly lead to dramatic phenotypes such

\section{贷 Springer}

(c) 2015 Johnson et al. This article is distributed under the terms of the Creative Commons Attribution 4.0 International License (http://creativecommons.org/licenses/by/4.0/), which permits unrestricted use, distribution, and reproduction in any medium, provided you give appropriate credit to the original author(s) and the source, provide a link to the Creative Commons license, and indicate if changes were made. 
as single giant chloroplasts covering entire mesophyll cells [8-10]. Z-ring formation/assembly depends on the balance between FtsZ and accessory chloroplast division proteins, which either stabilize (ARC6 [11, 12], MinE $[3,13,14]$ ) or destabilize (ARC3 [15], PARC6 [16]) FtsZ assemblies.

The properties and the assembly mechanism of plant FtsZ1 and FtsZ2 have been explored in vitro. FtsZ1 and FtsZ2 have differential guanosine triphosphatase (GTPase) activity $[17,18]$ and are capable of linear coassembly to form heteropolymers [17]. FtsZ1 and FtsZ2 exhibit dynamic turnover in vivo [19] that is promoted by the ARC3 protein [20]. How the individual FtsZ filaments assemble into higher-order structures to form the chloroplast dividing Z-ring is not well understood. Some insight has been gained from studies with FtsZ from prokaryotes. Cryo-TEM tomography revealed that the Z-ring is composed of short, partially overlapping protofilaments [21]. In contrast, in vitro reconstitution with only FtsZ and FtsA showed a continuous protofilament encircling the constricted liposomes several times [22]. The constriction force is thought to result from either bending of FtsZ protofilaments upon guanosine triphosphate (GTP) hydrolysis [23, 24] or, as was proposed recently, may involve protofilament sliding that is independent of GTP hydrolysis [22]. GTP hydrolysis and dynamic turnover are nevertheless essential for spatial regulation of Z-rings by accessory proteins that also mediate remodeling of FtsZ filaments into highly curved bundles and vortices $[25,26]$.

In contrast to bacterial FtsZ, the in vitro assembled plant FtsZ1 and FtsZ2 proteins did not show highly curved filaments $[17,27]$, hinting that in chloroplast the curvature and force for constriction could be generated by other components of the division machinery, such as the dynamin-related protein ARC5 residing on the outside of the chloroplast envelope $[28,29]$. The arrangement of FtsZ protofilaments in the Z-ring in chloroplasts and how they bend and proceed in chloroplast division remains unknown. Highly curved, small ring-like FtsZ assemblies, termed mini-rings, have been reported in plant chloroplasts and studied by optical microscopy [3, $15,30,31]$. It is hypothesized that the structural signature principles identified in these highly curved small assemblies may also play a role in the actual Z-ring. In this study, a detailed analysis of FtsZ mini-ring structure was conducted using SIM and electron tomography in Arabidopsis thaliana chloroplasts.

\section{Methods}

Plant material

Arabidopsis thaliana was chosen for this study as it is an established model organism. The $A$. thaliana ecotype
Columbia (Col-0) and the mutants used in this study were obtained from the Arabidopsis Biological Resource Center (ABRC; http://www.arabidopsis.org): arc6 (AT5G42480, CS286) and arc12 (At1G69390, CS16472). Negative control plants were untransformed ftsZ2-1/2-2 double knockout plants [10]. The AtFtsZ2-mYFP construct has been described previously [20]. Plants were grown in Redi-Earth (SunGro Horticulture, Bellevue, WA, USA) substrate in a rooftop greenhouse at a temperature of $72{ }^{\circ} \mathrm{C}$ and a relative humidity of $62 \%$. Agrobacterium-mediated transformation of Arabidopsis wild-type Col-0 plants and selection of the herbicide-resistant seedlings were performed as described $[4,32]$.

\section{Immunofluorescence labeling and optical microscopy}

Leaf and shoot meristematic tissue was fixed, embedded in Steedman's wax, immunolabeled with anti-FtsZ2 antibody and documented by wide-field fluorescence microscopy as described previously [4]. Live imaging was performed using an Olympus FV1000 confocal microscope (Olympus Scientific Solutions America, Waltham, MA, USA) equipped with a $60 \times / 1.2$ water immersion objective and a 515-nm laser for excitation. Prior to imaging, a drop of perfluorodecalin [33] was placed on the leaf tissue to remove air pockets from intercellular spaces and ensure good optical quality. YFP fluorescence was detected through a 535- to $575-\mathrm{nm}$ bandpass filter, while the chlorophyll autofluorescence was collected in the 670- to 700-nm range. Sections from resin-embedded tissue after HPF-FS were mounted in immersion oil and imaged using a $100 \times / 1.4$ oil immersion objective.

For structured illumination microscopy, leaf tissue was formaldehyde fixed under microwave irradiation, a process that preserves both the structural detail and the fluorescent protein signal $[20,34]$. The tissue was vacuum infiltrated with $3 \%$ formaldehyde in phosphatebuffered saline (PBS, 0.14 M NaCl, $2.7 \mathrm{mM} \mathrm{KCl,} 6.5 \mathrm{mM}$ $\mathrm{Na}_{2} \mathrm{HPO}_{4}, 5 \mathrm{mM} \mathrm{KH} \mathrm{PO}_{4}, 3.0 \mathrm{mM} \mathrm{NaN}$; $\left.\mathrm{pH}=7.3\right)$ and incubated at room temperature for $30 \mathrm{~min}$. It was then fixed in a Pelco Biowave (Ted Pella, Inc., Redding, CA, USA) laboratory microwave processor equipped with a ColdSpot $^{\circledR}$ temperature control system, with power set to $250 \mathrm{~W}$ and a 6 -min cycle $(2 \mathrm{~min}$ on, $2 \mathrm{~min}$ off, $2 \mathrm{~min}$ on). The temperature cutoff was set to $37{ }^{\circ} \mathrm{C}$. The tissue was then rinsed in PBS three times, with microwave irradiation $1 \mathrm{~min}$ at $250 \mathrm{~W}$ power in each rinse and then gradually infiltrated with 30, 50, $80 \% \mathrm{v} / \mathrm{v}$ Glycerol in PBS mixture, with microwave irradiation of $1 \mathrm{~min}$ at $250 \mathrm{~W}$ power in each step. The tissue was then left in fresh $80 \%$ v/v glycerol/PBS at $4{ }^{\circ} \mathrm{C}$ overnight and then either sent for imaging on the OMX microscope to GE Healthcare (formerly Applied Precision, Inc. Issaquah, WA, USA) or imaged on a Zeiss Elyra-S system. OMX imaging was 
performed using a $100 \times / 1.4$ oil immersion objective, excitation $488 \mathrm{~nm}$, emission centered at $525 \mathrm{~nm}$, and voxel size $40 \times 40 \times 120 \mathrm{~nm}$. Elyra-S imaging was performed with a $63 \times / 1.4$ oil immersion objective and voxel size $40 \times 40 \times 110 \mathrm{~nm}$. Mini-ring diameter was measured using ImageJ software (http://imagej.nih.gov/ij/) for mini-rings that were parallel to the XY plane. A line intensity profile across the mini-ring was plotted and the peak-to-peak distance, which corresponds to mini-ring diameter, was determined from these plots.

\section{Immunoblotting}

Extracts from expanding leaves from approximately 5 -week old plants were prepared, separated by SDSPAGE and analyzed by immunoblotting as described [35]. Extract from approximately 1-mg fresh tissue was loaded per lane. Immunodetection with affinity-purified goat anti-peptide antibody, recognizing the residues 168 through 184 in AtFtsZ2-1 [35] was performed as described in Johnson et al. [20]. Equal loading was confirmed by Ponceau S staining of the RuBisCO band on membranes $(0.1 \%(\mathrm{w} / \mathrm{v})$ Ponceau $\mathrm{S}$ in $5 \%(\mathrm{v} / \mathrm{v})$ acetic acid).

\section{High-pressure freezing (HPF)-freeze substitution (FS)}

Transgenic plants 3-4 weeks old were prescreened using a laser scanning confocal microscope for the presence of FtsZ mini-rings prior to HPF. A small piece of leaf tissue from either a plant expressing a FtsZ2-mYFP or from a control plant lacking FtsZ2 proteins was extracted from the base of young $(20-30 \mathrm{~mm})$ leaves using either a 2-mm-diameter biopsy punch or a scalpel and loaded into a Type B 0.3-mm deep specimen carrier (Technotrade, Manchester, NH, USA) filled with the cryoprotectant, 1-hexadecene (Sigma-Aldrich, Saint Louis, MO, USA). The filled specimen carrier was covered with the flat side of another specimen carrier. Subsequently, the specimen carrier sandwich was immediately loaded into a specimen carrier holder, inserted into the Wohlwend Compact 01 HPF machine (Technotrade, Manchester, NH, USA) and cryoimmobilized at $-190{ }^{\circ} \mathrm{C}$ and $204.5 \mathrm{MPa}$ of pressure. Afterward, the specimen holder was rapidly removed from the HFP machine and plunged into a liquid nitrogen $\left(\mathrm{LN}_{2}\right)$-filled chamber. The specimen carriers containing the frozen hydrated samples were transferred under $\mathrm{LN}_{2}$ to the pre-chilled cryovials using pre-cooled tweezers and stored in $\mathrm{LN}_{2}$ until further processing. The FS solution used on the HFP samples was $4 \%(\mathrm{w} / \mathrm{v})$ uranyl acetate (UA) dissolved in glass-distilled acetone (EMS, Hatfield, PA, USA) that was opened immediately prior to use. Steps in the conventional as well as the quick freeze substitution (QFS) procedure are outlined in Table 1. The QFS method
Table 1 Conventional FS and modified QFS procedures

\begin{tabular}{ll}
\hline Conventional FS & Modified QFS \\
\hline FS (4 \% UA/acetone) & FS (4 \% UA/acetone) \\
$-90^{\circ} \mathrm{C}(76 \mathrm{~h})$ & -169 to $23^{\circ} \mathrm{C}$ over the course of $5 \mathrm{~h}$ \\
$-45^{\circ} \mathrm{C}(30 \mathrm{~h})$ & \\
Infiltration schedule $\left(-25^{\circ} \mathrm{C}\right)$ & Infiltration schedule $\left(25^{\circ} \mathrm{C}\right)$ \\
Lowicryl-HM20/acetone & LRWhite/acetone \\
$10 \%(6 \mathrm{~h})$ & $10 \%(13 \mathrm{~h})$ \\
$25 \%(16 \mathrm{~h})$ & $20 \%(3 \mathrm{~h})$ \\
$50 \%(7.5 \mathrm{~h})$ & $30 \%(2 \mathrm{~h})$ \\
$75 \%(14.5 \mathrm{~h})$ & $40 \%(19 \mathrm{~h})$ \\
$100 \%(6.5,19,7 \mathrm{~h})$ & $50 \%(2 \mathrm{~h})$ \\
& $60 \%(2 \mathrm{~h})$ \\
& $70 \%(3 \mathrm{~h})$ \\
& $80 \%(1 \mathrm{~h})$ \\
& $90 \%(1 \mathrm{~h})$ \\
& $100 \%(15,2,2 \mathrm{~h})$ \\
$-45^{\circ} \mathrm{C}(48 \mathrm{~h})$ & Polymerization \\
\hline $0^{\circ} \mathrm{C}(48 \mathrm{~h})$ & $60{ }^{\circ} \mathrm{C}(24 \mathrm{~h})$ \\
\hline
\end{tabular}

UA uranyl acetate, FS freeze substitution

is a modification of the protocol that was first described by McDonald and Webb [36].

\section{Microtomy and immunogold labeling}

Semi-thin $(300 \mathrm{~nm})$ sections were cut on a Reichert-Jung Ultracut $\mathrm{E}$ microtome equipped with a diamond knife and mounted onto silane-coated microscope slides for light microscopy. Thin sections $(80-100 \mathrm{~nm})$ were cut in the same manner and picked up on uncoated nickel grids. Thin sections were blocked with $4 \%(\mathrm{v} / \mathrm{v})$ cold water fish gelatin (Sigma) in PBS in the Biowave microwave processor with power set to $250 \mathrm{~W}$ and a $2-2-2$ cycle $(2 \mathrm{~min}$ on, $2 \mathrm{~min}$ off, $2 \mathrm{~min}$ on). The temperature cutoff was set to $37^{\circ} \mathrm{C}$. The grids were reacted with monoclonal mouse anti-GFP antibody (Millipore, Temecula, CA, USA) diluted 1:500 in the PBS blocker, using the 2-2-2 cycle. Grids were washed $2 \times 1$ min with PBS and $2 \times 1 \mathrm{~min}$ with Tris-buffered saline (TBS; $0.15 \mathrm{M} \mathrm{NaCl}, 20 \mathrm{mM}$ Tris- $\mathrm{HCl} \mathrm{pH} \mathrm{7.4),} \mathrm{then} \mathrm{blocked} \mathrm{with} 4 \%$ (v/v) cold water fish gelatin in TBS at $250 \mathrm{~W}$ using the $2-2-2$ cycle. Grids were incubated in a 1:30 dilution of donkey anti-mouse IgG conjugated to 12-nm colloidal gold (Jackson ImmunoResearch, West Grove, PA, USA) at $250 \mathrm{~W}$ with a 2-2-2 cycle. Grids were washed in TBS $3 \times 1 \mathrm{~min}$ followed by $3 \times 1 \mathrm{~min}$ with deionized water, then reacted with $1 \%(\mathrm{v} / \mathrm{v})$ glutaraldehyde for $5 \mathrm{~min}$ on the bench before being washed for $3 \times 1$ min with deionized water and dried on a slide warmer. 
Transmission electron microscopy and tomography

Grids were viewed in an FEI (Hillsboro, OR, USA) Tecnai $^{\text {TM }}$ G2 F20 operated at an acceleration voltage of $200 \mathrm{kV}$ and equipped with a dedicated Fischione (Export, PA, USA) high-angle annular dark-field (HAADF) STEM detector, a GATAN (Pleasanton, CA, USA) Tridiem energy filter and a $2 \mathrm{k} \times 2 \mathrm{k}$ GATAN Ultrascan 1000 CCD camera. Micrographs were recorded at calibrated magnifications, leading to a sampling of $1.1 \mathrm{~nm} /$ pixel in STEM and $0.39 \mathrm{~nm} /$ pixel in energy-filtered TEM mode. Tilt series were acquired from $-75^{\circ}$ to $+73^{\circ}$ in $1^{\circ}$ increments using the FEI Xplore $3 \mathrm{D}^{\mathrm{TM}}$ software. Datasets were reviewed using FEI Inspect 3D.

\section{Reconstruction, segmentation and 3D rendering}

All processing was performed in EM3D [37, 38]. Micrographs from $-50^{\circ}$ to $50^{\circ}$ tilt could effectively be used yielding a total of 101 projections. Where gold fiducials were not present, alignment was done using statistically consistent features in the background as fiducial markers. Aligned projections were reconstructed into a 3D volume with 72 slices. The object edge on each slice was defined by dots, and the spline option provided a smooth edge for the connected dots. With filaments extending over 8-20 slices and with stacked regions, sudden changes in features were defined as edges between filaments. Segmented protofilaments were then colored and rendered with $40 \%$ saturation of isosurface value so as not to smooth out surface modulations. Curvature measurements were performed in ImageJ with the three-point circular ROI plugin. The curvature was calculated as the inverse of the circle radius $(1 / r)$.

Images for publication were adjusted for contrast using Gimp 2.8. freeware (http://www.gimp.org) and multipanel figures assembled using Scribus ver. 1.4.2. freeware (http://www.scribus.net).

\section{Results and discussion \\ FtsZ rings and mini-rings}

In wild-type mature plants, consistent with previous reports [4, 9, 39], immunofluorescence labeling with an anti-FtsZ2 antibody showed FtsZ rings (Z-rings) encircling the mid-chloroplast in leaf mesophyll cells (Fig. 1a). Meristematic cells that harbor smaller plastids or proplastids contained much smaller Z-rings (Fig. 1b) that also presumably encircled the plastid, but the shape of these small plastids could not be determined from the micrographs. Another type of FtsZ assembly, small Z-rings, was often encountered in leaf mesophyll chloroplasts under conditions described below. These circular FtsZ assemblies are not restricted to the mid-plastid, their size is not dictated by the diameter of the plastid and they measure reproducibly and significantly less than $1 \mu \mathrm{m}$ in diameter. Some are so small that they are only resolved as puncta in a standard light microscope., i.e., their diameter is $\leq 250 \mathrm{~nm}$. These small circular FtsZ assemblies have been termed FtsZ mini-rings or mini-rings for short. The conditions under which they are formed are varied and complex. Mini-rings have for instance been observed in Arabidopsis wild-type background, moderately expressing FtsZ2-mYFP (Fig. 1c), wild-type background plants overexpressing FtsZ2mYFP (Fig. 1d) or in mutants lacking the Z-ring-stabilizing factors ARC6 [31] or MinE [30] (Fig. 1e, f). Figure 1e, $\mathrm{f}$ is particularly relevant as native, untagged FtsZ was detected by a fluorescently labeled antibody. This means that min-ring formation need not be considered an artifact of fluorescent protein tagging.

\section{Characterization of mini-rings by superresolution light microscopy}

SIM capable of analyzing samples at a resolution beyond the Abbe limit [40] was chosen for a more detailed analysis of mini-rings in FtsZ2-mYFP overexpressing plants. The resolution of SIM images was estimated by measuring full width at half maximum (FWHM) \pm SD of linear FtsZ filaments. Data from the OMX microscope provided a resolution of $118 \pm 11 \mathrm{~nm}(\mathrm{n}=28)$ laterally and $354 \pm 26 \mathrm{~nm}(\mathrm{n}=27)$ axially. Ringing artifacts were noted, probably due to aggressive image reconstruction. Resolution in the Zeiss Elyra-S images was $138 \pm 13 \mathrm{~nm}$ $(\mathrm{n}=29)$ laterally and $547 \pm 70 \mathrm{~nm}(\mathrm{n}=25)$ axially with less pronounced processing artifacts. The latter images also contained chlorophyll fluorescence channel suitable for measurement of chloroplast size and volume. Nearly, all of the small punctate structures were resolved as minirings (Fig. 2a) with a mean diameter of $208 \pm 68 \mathrm{~nm}$ $(\mathrm{n}=248)$ (Fig. $2 \mathrm{~b})$. The mean chloroplast volume was determined as $660 \pm 450 \mu^{3}(\mathrm{n}=20)$ containing between 10 and 16 rings in most cases with an occasional surge up to $40-80$. The increased resolution afforded by SIM images allowed to resolve the mini-rings below the diffraction limit and to investigate the spatial relationship between circular and linear FtsZ assemblies. It was noted that while some $(32.2 \% ; \mathrm{n}=214)$ mini-rings appeared to be isolated from other FtsZ assemblies, most (67.8 \%; $\mathrm{n}=214$ ) appeared to be terminally attached to FtsZ filaments. Examples of mini-ring attachments to filaments are shown in Fig. 2c. The attachment of mini-rings to linear FtsZ filaments was previously observed by (1) immunofluorescence labeling in the arc6 mutant (S. Vitha, unpublished), (2) in vivo time-lapse microscopy in the Arabidopsis minE mutant [30] and (3) in yeast S. pombe expressing bacterial FtsZ-GFP [41]. It has been proposed that the mini-rings are formed by spooling the end of single or multiple filaments [30, 41]. 

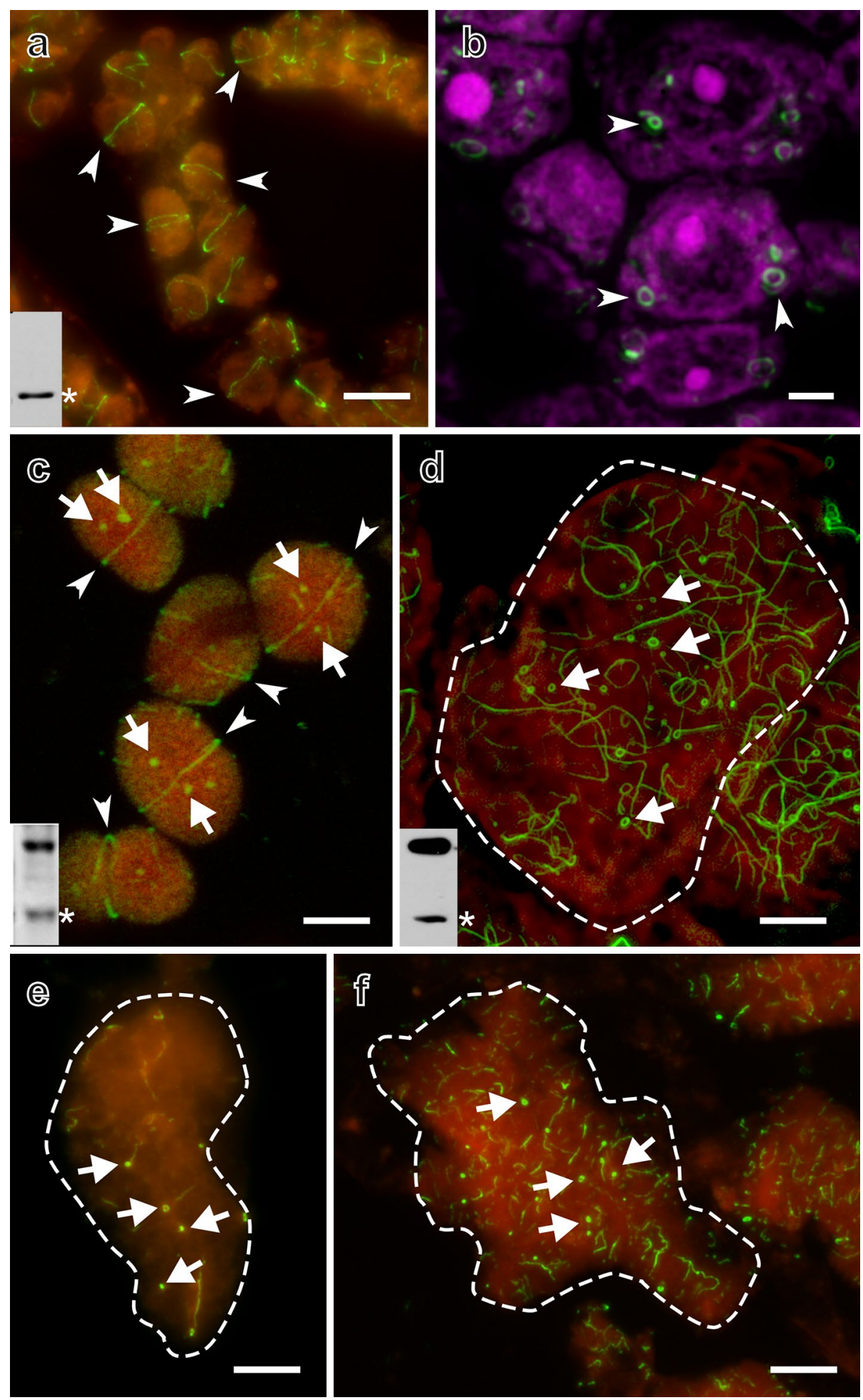

Fig. 1 Fluorescence microscopy of FtsZ2 assemblies in situ. a, b Wide-field immunofluorescence micrograph of FtsZ2 in Z-rings (arrowheads) in wild-type leaf chloroplasts (a) and smaller-size Z-rings in cells of the apical meristem (b). Individual proplastids cannot be discerned in meristematic cells. c, d Confocal micrographs of filamentous and circular (arrows) FtsZ assemblies in leaf mesophyll chloroplasts in a plant expressing FtsZ2-mYFP at moderate (c) and high (d) levels. The arrowheads in c denote Z-rings at mid-chloroplast. Inserts in $\mathbf{a}, \mathbf{c}$, $\mathbf{d}$ depict immunoblots showing relative levels of the intrinsic, untagged FtsZ2 (marked by an asterisk) vs. FtsZ2-fusion protein. e, f Immunofluorescence localization of FtsZ2 in leaf mesophyll chloroplasts of mutants lacking MinE and ARC6 proteins, respectively. FtsZ mini-rings are indicated by arrows. The dashed lines in $\mathbf{d}-\mathbf{f}$ denote chloroplast shapes. Scale bars correspond to $5 \mu \mathrm{m}$ in $\mathbf{a}, \mathbf{c}-\mathbf{f}$ and $2 \mu \mathrm{m}$ in $\mathbf{b}$ 

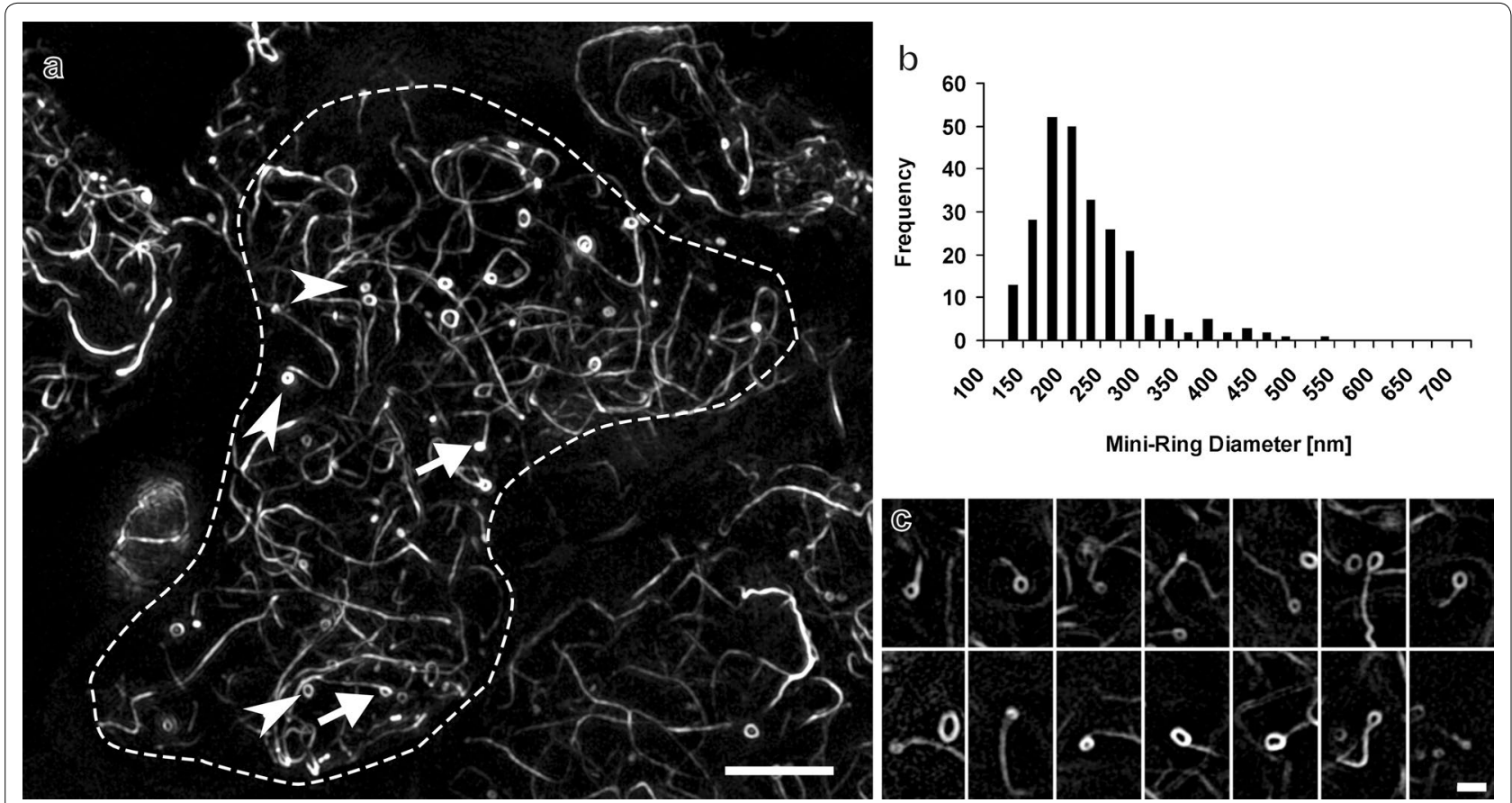

Fig. 2 SIM imaging of FtsZ2-mYFP mini-rings and filamentous assemblies in leaf mesophyll chloroplasts. a Maximum intensity projection showing both filaments and mini-rings in an enlarged leaf chloroplast. The dashed line delineates the shape of the chloroplast. Some mini-rings appear attached to the end of linear FtsZ filaments (arrows), while others are unattached (arrowheads). b Distribution of mini-ring diameters. c Optical sections showing FtsZ mini-rings contiguous with linear FtsZ filaments. Scale bars correspond to $5 \mu \mathrm{m}$ in $\mathbf{a}$ and $1 \mu \mathrm{m}$ in $\mathbf{c}$

\section{HPF-FS for correlative imaging of plant chloroplasts}

To investigate the mini-ring assemblies at macromolecular dimensions in situ, leaf samples had to be prepared for electron microscopy. The ability to study-specific molecular targets and their 3D organization by fluorescence microscopy and to subsequently explore the same object in detail by electron microscopy sidesteps the limitations of either technique. The challenge of plant tissue and especially the localization of fluorescent proteins inside chloroplast is quenching of the fluorescent protein signal and high tissue autofluorescence that can be induced during sample preparation. The requirements for sample preparation were (1) structural preservation, (2) maintaining specific fluorescence to aid correlative light and electron microscopy, and (3) minimizing the autofluorescence of the plant chloroplast. Initially, methods employing isolation of intact chloroplasts, and extraction and fractionation on density gradients [42] were explored to obtain chloroplast division machineries. These attempts needed to be eventually abandoned as they met with little to no success. Following on from this, microwave-assisted aldehyde fixation of leaf tissue followed by dehydration and resin embedding were pursued. While live leaf tissue before embedding (Fig. 3a) was characterized by an excellent signal-to-noise ratio showing several mini-rings per chloroplast, semi-thin section of embedded tissue exhibited a loss of YFP fluorescence in combination with a substantial increase in autofluorescence background (Fig. 3b) preventing the detection of FtsZ2-mYFP assemblies. Ultimately, highpressure freezing in conjunction with freeze substitution (HPF-FS) and embedding in acrylic resins led to a successful preservation of the specific fluorescence signal as well as an attenuation of autofluorescence. The QFS procedure developed by McDonald and Webb [36] was adapted to introduce time and cost savings. In keeping with the main objectives, i.e., maintaining signal and minimizing fluorescence background, it was necessary to modify the infiltration procedure. This entailed changing the resin from Epon/acetone to LR White/acetone and extending the infiltration to a more gradual protocol (see "Methods"). FtsZ-mYFP fluorescence within chloroplasts was preserved and easily detected in semi-thin sections (300 nm), and a low autofluorescence background was maintained revealing both mini-rings and short linear FtsZ assemblies (Fig. 3c). The lack of chloroplast-localized FtsZ signals in sections from control tissue lacking FtsZ2 (Fig. 3d) confirmed that the signals observed in Fig. 3c were due to FtsZ2-mYFP.

The HPF approach offers to preserve organelles and other cellular components by instantaneous stabilization [43] in a manner superior to chemical fixatives [44]. 

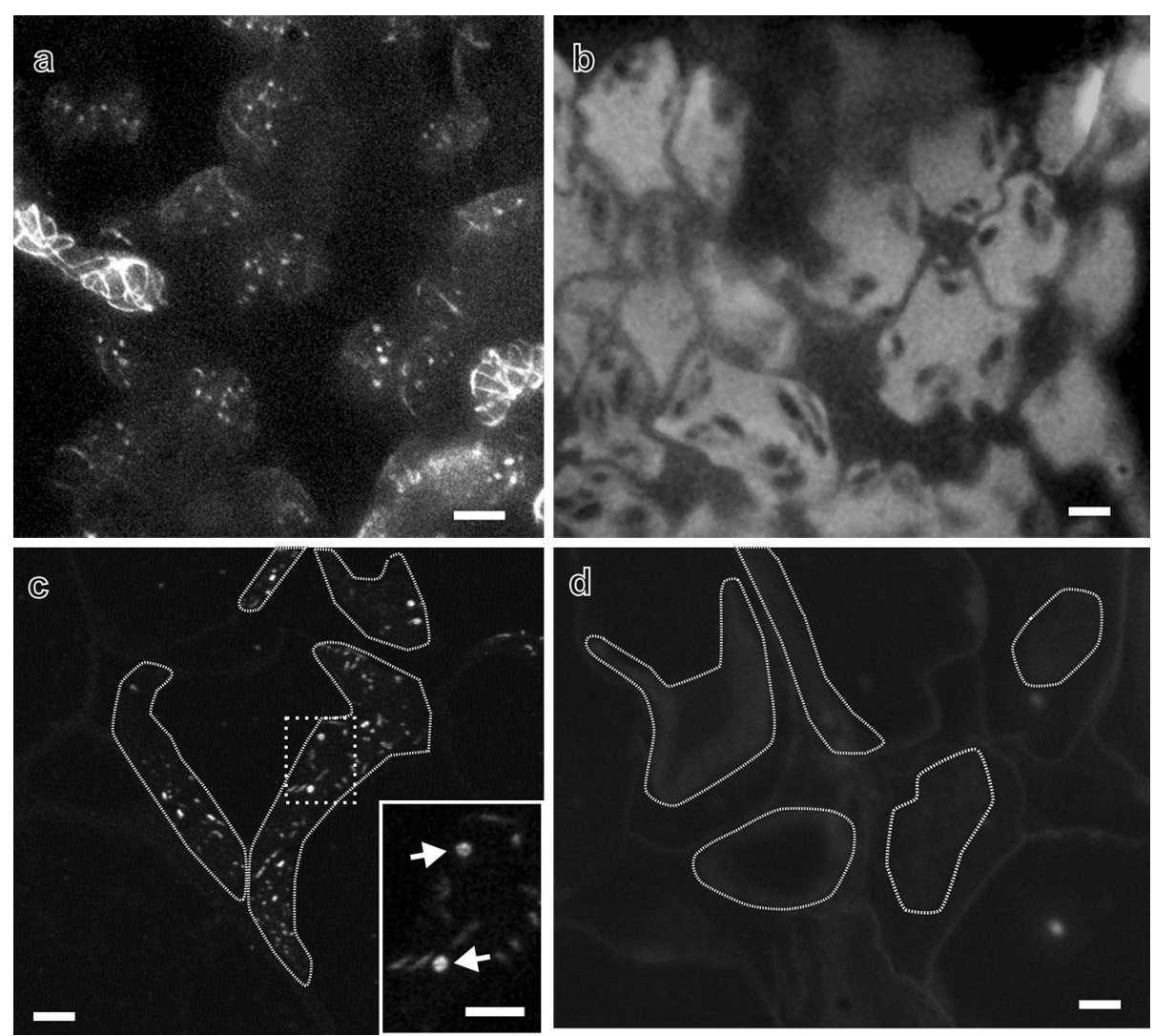

Fig. 3 Preservation of YFP fluorescence after resin embedding. a Wide-field fluorescence microscopy of live leaf tissue showing multiple FtsZ2mYFP mini-rings per chloroplast. b Semi-thin section from aldehyde-fixed tissue embedded in LR White. Chloroplasts are recognized by their high fluorescence background. c-d Semi-thin sections from leaf tissue subjected to HPF-FS and embedding in LR White. Chloroplasts are delineated by dotted lines. $\mathbf{c}$ Punctate and short linear FtsZ2-mYFP assemblies in resin sections. The area corresponding to the highlighted square is shown at higher magnification in the insert where some of the assemblies are resolved as mini-rings (arrows). $\mathbf{d}$ Mutant plant lacking FtsZ2 proteins (negative control). Scale bars represent $5 \mu \mathrm{m}$ in $\mathbf{a}-\mathbf{d}$ and $2 \mu \mathrm{m}$ in the insert in $\mathbf{c}$

Both acrylic resins used, Lowicryl HM20 and LR White, proved capable of maintaining the fluorescent protein signal. This is in agreement with previous reports where methacrylates, LR White or Lowicryl have been employed in conjunction with plant and animal tissue and yeast cells [45-48].

\section{In situ electron microscopy/tomography of chloroplast mini-ring assemblies}

Prior studies with prokaryotic FtsZ, which is closely related to the plant FtsZ2 protein [5-7], showed the presence of small circular assemblies under certain in vitro assembly conditions. Some were very small, measuring approximately $25 \mathrm{~nm}$ in diameter and were termed minirings $[49,50]$. Under conditions of molecular crowding, larger circular assemblies $(\sim 200 \mathrm{~nm})$ termed mini-rings or toroids were reported $[51,52]$. The relevance of these in vitro assemblies to the assembly of FtsZ in vivo is being debated.
In the current study, both STEM and TEM imaging of thin sections from resin-embedded tissue revealed small circular structures (Fig. 4a, b, respectively) with a mean diameter of $183 \pm 50 \mathrm{~nm}(\mathrm{n}=21)$, which is in excellent agreement with the mini-ring diameter distribution determined by SIM imaging (Fig. 2). In negative control samples from FtsZ2-null mutant Arabidopsis, no circular assemblies could be observed. While mini-rings presented themselves as 'solid' rings by conventional light microscopy as well as SIM imaging, much more complex features were discernible by TEM and STEM. The rings appeared to be made up of multiple strands and bundles of various diameters and different degrees of order (Fig. 4). In particular, it was noted that roughly half of each ring was 'tight', while the other half appeared somewhat frayed. In the STEM dark-field image (Fig. 4a), because of the higher contrast when compared with the TEM bright-field image (Fig. 4b), even individual strands in a parallel packing arrangement between 10 and 12 

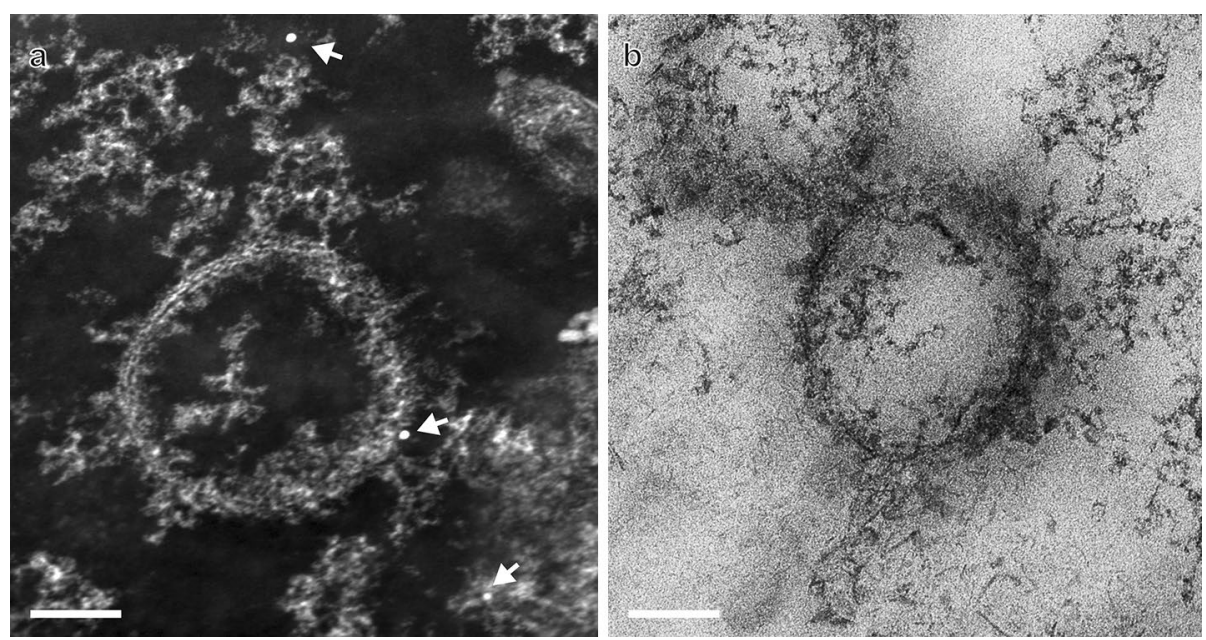

Fig. 4 STEM and TEM of mini-ring assemblies in thin sections. Single images from a tomographic tilt series are shown. a STEM dark-field image with 12-nm gold fiducial markers indicated by arrows. b TEM bright-field image depicting a different mini-ring. Scale bars correspond to $100 \mathrm{~nm}$

o'clock are readily discernible. To further decipher the intrinsic architecture of these mini-rings and improve the signal-to-noise ratio, tomography in conjunction with $3 \mathrm{D}$ reconstruction was employed. With only $10-16$ mini-rings per chloroplast (see above) and one thin section of approximately $100 \mathrm{~nm}$ (the thickness of a chloroplast is between 5 and $10 \mu \mathrm{m}$ corresponding to $50-100$ thin sections) needing to harbor a mini-ring that is located sufficiently central in a grid window not affected by grid bars upon tilting the specimen and aligned parallel with the plane of the thin section, tomographycompatible mini-rings were rarely encountered. While the criteria for extended slab geometry in tomographic reconstructions suggests in this case an isotropic 3D resolution of only around $4-5 \mathrm{~nm}$, given the tilt angle range, thickness and number of projections [53], the resolution of a single section in projection is less constrained by these parameters and thus may reveal detail not readily available in the $3 \mathrm{D}$ rendering. This is demonstrated in the section of a tomogram shown in Fig. 5. Surveying the overall structural revealed a region with clearly separated strands as highlighted by the boxed area (Fig. 5a). Further magnifications of the area of interest (Fig. 5b, c) show clearly discernible linearly arranged densities measuring $12.6 \mathrm{~nm}$ over three repeats making one repeat $4.2 \mathrm{~nm}$ along the axis and approximately $3 \mathrm{~nm}$ perpendicular to it (strand thickness). Comparing the observed density distribution with the overall structural characteristics of an FtsZ protofilament (Fig. 5e, f) suggests that the observed strands correspond to protofilaments. While single sections may show more details for small regions of interest like those highlighted area in Fig. 5, other regions in the mini-ring appear very disordered, such as the entire right-hand side of the mini-ring. Here, clearly discernible features may only stand out by taking the entire 3D information into consideration. This was achieved by tracking individual protofilaments through 3D space and subjecting the tomograms to segmentation, the results of which are shown in Fig. 6. A previous tomography study revealed segments of FtsZ protofilaments during bacterial cell division and showed that the Z-ring was composed of multiple parallel, relatively short and overlapping protofilaments [21]. The present data reveal that the FtsZ mini-ring has a more complex, multilayered structure composed of many protofilaments: 49 have been counted for the mini-ring shown (Fig. 6a).

On comparing the left-hand side (LHS) with the right-hand side (RHS) of the ring, different degrees of orders are observed echoing the trend already observed before segmentation and rendering (Figs. 4, 5). The RHS shows a higher degree of unraveling and entanglement, while the LHS is characterized by one curvilinear bundle consisting of parallel protofilaments surrounded by loosely interwoven filaments. Another distinct difference between the LHS and RHS relates to overall filament curvature. While the RHS of the ring showed only a slight degree of curvature, the LHS' curvature was on average threefold higher (Fig. 6b). This suggests a correlation between protofilament bundling and curvature. Upon closer inspection of the $3 \mathrm{D}$ reconstruction, it appears that the protofilaments can participate in higher-order structures at three levels. (1) The primary level is characterized by the aforementioned protofilaments. Distinct grooves delineate the long axis of the protofilaments at around 4-nm 


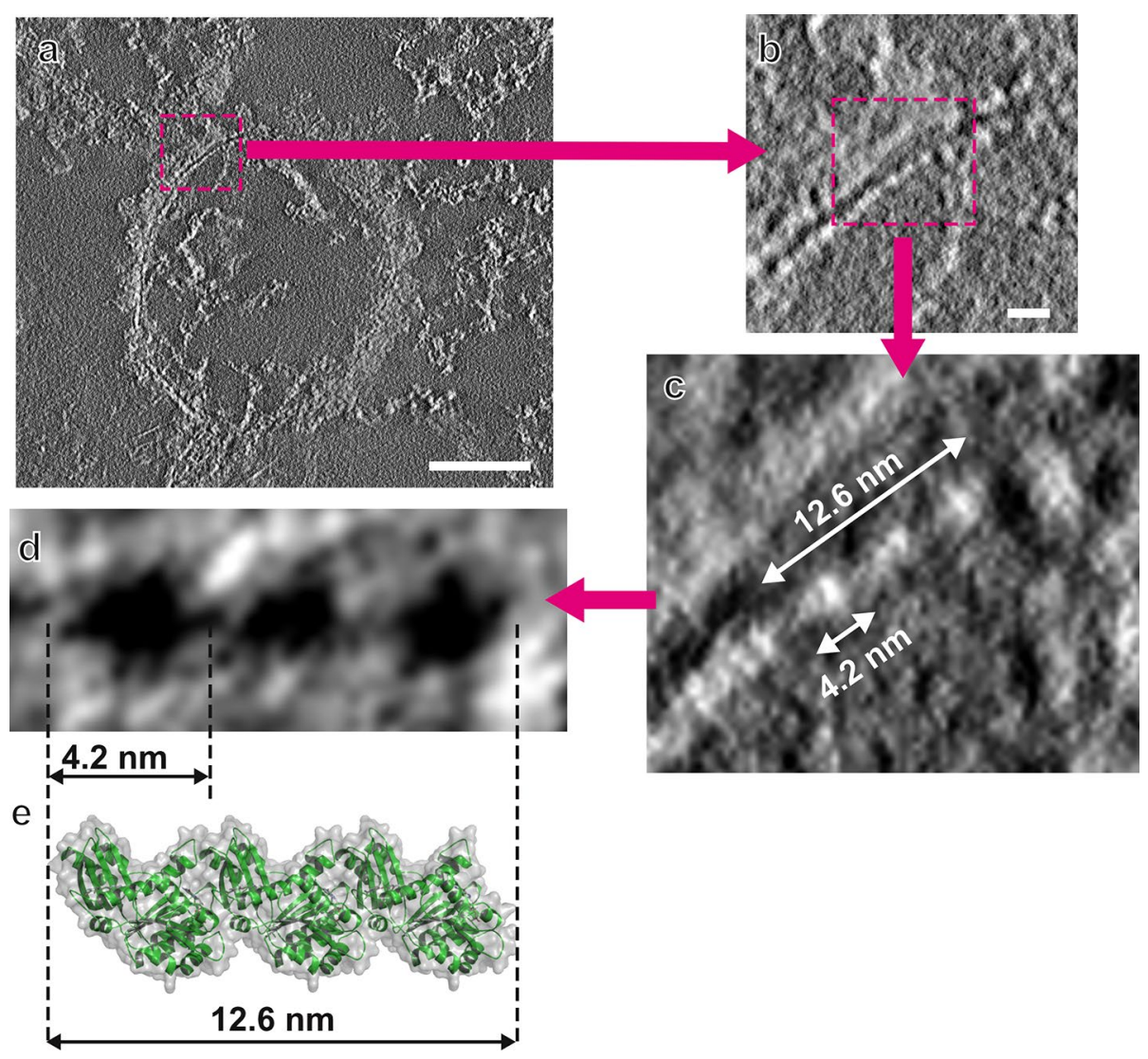

Fig. 5 Molecular details in an FtsZ mini-ring strand. a Central section from a 3D tomographic reconstruction of an in situ mini-ring revealing individual filamentous strands. b, c Zooming into the areas highlighted shows periodic densities as part of an individual strand. $\mathbf{d}$ Contrast-inverted final enlargement of three periodic densities extending over $12.6 \mathrm{~nm}$ with a $4.2-\mathrm{nm}$ repeat. Length scale, periodicity and shape are in excellent agreement with the $X$-ray structure of an FtsZ protofilament in $\mathbf{e}$. Fitted atomic structures of individual FtsZ subunits are shown in green. Scale bars correspond to $100 \mathrm{~nm}$ in $\mathbf{a}$ and $10 \mathrm{~nm}$ in $\mathbf{b}$

intervals and these filaments measure approximately $3 \mathrm{~nm}$ across (Fig. 6c). (2) Secondary-level structures are represented by two protofilaments together twisting around a central axis, thus creating quasi-double helical structures. These structures have a pitch of between 10 and $15 \mathrm{~nm}$ and a width of $7.6 \mathrm{~nm}$ (Fig. 6d). These data are in good agreement with the $13.25-\mathrm{nm}$ pitch observed with double-stranded FtsZ protofilaments from Mycobacterium tuberculosis [21]. (3) A tertiary level of hierarchical structures is observed when double helical structures intertwine into superhelical assemblies, the example of which is depicted in Fig. 6e. As a general trend for this particular mini-ring, the higher-order structures dominate the RHS of the ring while the more orderly structured LHS appears to be more populated by protofilaments. Such a dichotomy points at a dynamics not reported previously. In essence, the structure looks as if it presents a balance between two states: a state of disassembly and one of assembly. The latter could lead from an interwoven and entangled arrangement to orderly layered structures. We speculate that if the tightly arranged filament bundles are disturbed, individual filaments may be able to assume different higher-order structures such as the ones described above.

While the mini-rings could be considered metastable as per the earlier reasoning, they may also serve in this capacity as an energetically favorable way of protofilament storage. This would be particularly important in times of high abundance of FtsZ such as that observed in young, metabolically active cells. By virtue of their curvature, they could also bestow the assembly with inherent energy leading to pre-energized, 'ready-to-assemble' protofilaments for a more responsive supply of Z-ring building blocks. The relatively high percentage of minirings found attached to linear filaments corroborate such an idea.

In a broader context, the presented data provide first insights into the molecular architecture of plant FtsZ assemblies in situ featuring bundling of filaments, a 

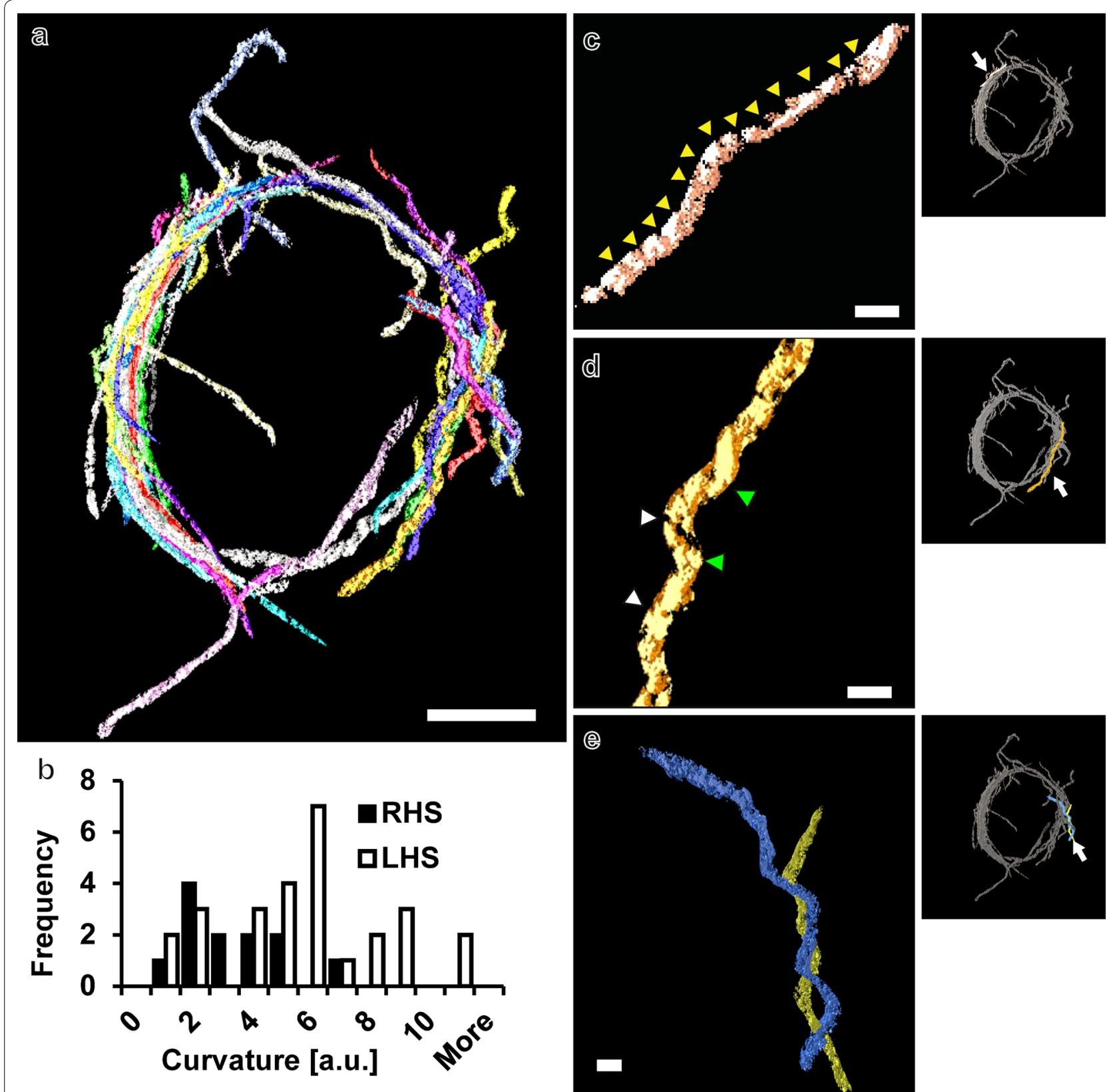

Fig. 6 Mini-ring after 3D reconstruction and segmentation. a Overall organization of the FtsZ mini-ring with individual strands shown in different colors. b Distribution of protofilament curvature measured in the left- and right-hand side of the mini-ring (LHS and RHS, respectively). c A protofilament with 4- to 6-nm longitudinal repeats highlighted by yellow arrowheads. d Twisted filaments (double helix) with a pitch between 10 and $15 \mathrm{~nm}$. White and green arrowheads are separated by $360^{\circ}$ each, i.e., one turn along the central axis. e Intertwined superhelices. In c-e, the accompanying panels highlight the position of the featured objects (marked by an arrow) in the mini-ring. Scale bars correspond to $100 \mathrm{~nm}$ in $\mathbf{a}, 5 \mathrm{~nm}$ in $\mathbf{c}$ and $10 \mathrm{~nm}$ in $\mathbf{d}, \mathbf{e}$

multilayered structure, differential protofilament curvature as well as higher-order structures such as superhelices. While the curvature of plant FtsZ protofilaments was not observed in vitro $[17,27]$, clearly the mini-rings show that FtsZ is able to form curved assemblies without depending on cytosolic components such as ARC5 [28, 29]. Perhaps, the propensity of FtsZ to curve is predicated on the features summarized above. On the other hand, these features could also echo the dynamic remodeling that FtsZ assemblies are constantly undergoing $[19,20]$. 


\section{Conclusions}

1. Chloroplast division is highly complex and of societal importance as the mechanisms underlying this process also control the starch granule size.

2. FtsZ is a key protein of chloroplast division and this report presents the first electron tomographic 3D structure of plant FtsZ assembly in situ. The assembly form presented here are the FtsZ mini-rings which occur under defined physiological conditions.

3. Comparative SIM and EM imaging yielded matching mini-ring diameters. Combining light microscopy screening with a detailed EM analysis via tomography laid the foundations for the development of correlative microscopy-compliant protocols involving HPF and FS.

4. Assessment of resolution for the tomographic data set according to the Crowther theorem [53] only suggests 4 to $5-\mathrm{nm}$ resolution in the $3 \mathrm{D}$ reconstruction; however, the resolution is much better in projection as demonstrated by a comparison with X-ray data. The latter led to the identification of protofilaments in the tomogram. These protofilaments can also assemble into higher-order structures such as double helices and superhelical arrangements.

5. The data provide a glimpse into the dynamic life of FtsZ assemblies. Electron microscopy and tomography readily revealed a very dynamic picture of the mini-rings highlighted by a bipartite structure splitting the ring into an ordered and less ordered half. This implies that the rings may undergo dynamic remodeling by providing FtsZ building blocks for Z-ring assembly or a form of storage of protofilaments.

\section{Authors' contributions}

CBJ carried out the cloning, plant transformation and sample preparation for TEM, participated in the design of experiments and drafted the manuscript. ZLo performed the reconstruction and segmentation of the EM tomograms. ZLu performed the STEM and EFTEM imaging. RS carried out the immunoassays and MWS the fitting of/comparison with X-ray data. SV performed the confocal microscopy and carried out sample preparation for SIM. AH conceived the study and participated in EM data analysis. AH and SV participated in the design of experiments and carried out the SIM data analysis. AH and SV co-wrote the manuscript. All authors read and approved the final manuscript.

\section{Author details}

${ }^{1}$ Department of Biology, Texas A\&M University, College Station, TX 77843-3258, USA. ${ }^{2}$ Department of Chemistry and Biochemistry, University of California San Diego, La Jolla, CA 92093, USA. ${ }^{3}$ Department of Chemistry and Physics, Fayetteville State University, Fayetteville, NC 28301, USA. ${ }^{4}$ Microscopy and Imaging Center, Texas A\&M University, Interdisciplinary Life Sciences Building, 2257 TAMU, College Station, TX 77843-2257, USA. ${ }^{5}$ Department of Biochemistry and Biophysics, Texas A\&M University, College Station, TX 77843-3258, USA.

\section{Acknowledgements}

The authors thank the Office of the Vice President for Research at Texas A\&M University for the continued support of the Microscopy and Imaging Center. SIM was performed by GE Healthcare Life Sciences (formerly Applied Precision; Issaquah, WA, USA) and Zeiss Microimaging (Thornwood, NJ, USA).
The fts $Z 2-1 / 2-2$ double knockout plants were obtained from Dr. Katherine Osteryoung (Michigan State University).

\section{Compliance with ethical guidelines}

\section{Competing interests}

The authors declare that they have no competing interests.

Received: 28 July 2015 Accepted: 28 August 2015

Published online: 17 September 2015

\section{References}

1. Lindeboom, N., Chang, P.R., Tyler, R.T.: Analytical, biochemical and physicochemical aspects of starch granule size, with emphasis on small granule starches: a review. Starch Starke 56(3-4), 89-99 (2004)

2. de Pater, S., Caspers, M., Kottenhagen, M., Meima, H., ter Stege, R., de Vetten, N.: Manipulation of starch granule size distribution in potato tubers by modulation of plastid division. Plant. Biotech. J. 4(1), 123-134 (2006)

3. Maple, J., Aldridge, C., Møller, S.G.: Plastid division is mediated by combinatorial assembly of plastid division proteins. Plant J. 43(6), 811-823 (2005)

4. Vitha, S., McAndrew, R.S., Osteryoung, K.W.: FtsZ ring formation at the chloroplast division site in plants. J. Cell Biol. 153(1), 111-119 (2001)

5. Miyagishima, S.Y., Nozaki, H., Nishida, K., Nishida, K., Matsuzaki, M., Kuroiwa, T.: Two types of FtsZ proteins in mitochondria and red-lineage chloroplasts: the duplication of FtsZ is implicated in endosymbiosis. J. Mol. Evol. 58(3), 291-303 (2004)

6. Stokes, K.D., Osteryoung, K.W.: Early divergence of the FtsZ1 and FtsZ2 plastid division gene families in photosynthetic eukaryotes. Gene $\mathbf{3 2 0}$ 97-108 (2003)

7. Terbush, A.D., Yoshida, Y., Osteryoung, K.W.: FtsZ in chloroplast division: structure, function and evolution. Curr. Opin. Cell Biol. (2013). doi:10.1016/j.ceb.2013.04.006

8. Osteryoung, K.W., Stokes, K.D., Rutherford, S.M., Percival, A.L., Lee, W.Y.: Chloroplast division in higher plants requires members of two functionally divergent gene families with homology to bacterial ftsZ. Plant Cell 10(12), 1991-2004 (1998)

9. Yoder, D.W., Kadirjan-Kalbach, D., Olson, B.J.S.C., Miyagishima, S.Y., DeBlasio, S.L., Hangarter, R.P., Osteryoung, K.W.: Effects of mutations in Arabidopsis FtsZ1 on plastid division, FtsZ ring formation and positioning, and FtsZ filament morphology in vivo. Plant Cell Physiol. 48(6), 775-791 (2007)

10. Schmitz, A.J., Glynn, J.M., Olson, B.J.S.C., Stokes, K.D., Osteryoung, K.W.: Arabidopsis FtsZ2-1 and FtsZ2-2 are functionally redundant, but FtsZbased plastid division is not essential for chloroplast partitioning or plant growth and development. Mol. Plant 2(6), 1211-1222 (2009). doi:10.1093/ $\mathrm{mp} / \mathrm{ssp} 077$

11. Glynn, J.M., Froehlich, J.E., Osteryoung, K.W.: Arabidopsis ARC6 coordinates the division machineries of the inner and outer chloroplast membranes through interaction with PDV2 in the intermembrane space. Plant Cell 20(9), 2460-2470 (2008)

12. Vitha, S., Froehlich, J.E., Koksharova, O., Pyke, K.A., van Erp, H., Osteryoung, K.W.: ARC6 Is a J-domain plastid division protein and an evolutionary descendant of the cyanobacterial cell division protein Ftn2. Plant Cell 15(8), 1918-1933 (2003)

13. Maple, J., Chua, N.H., Møller, S.G.: The topological specificity factor AtMinE1 is essential for correct plastid division site placement in Arabidopsis. Plant J. 31(3), 269-277 (2002)

14. Fujiwara, M.T., Hashimoto, H., Kazama, Y., Abe, T., Yoshida, S., Sato, N., Itoh, R.D.: The assembly of the FtsZ ring at the mid-chloroplast division site depends on a balance between the activities of AtMinE1 and ARC11/ AtMinD1. Plant Cell Physiol. 49(3), 345-361 (2008)

15. Zhang, M., Schmitz, A.J., Kadirjan-Kalbach, D.K., Terbush, A.D., Osteryoung, K.W.: Chloroplast division protein ARC3 regulates chloroplast FtsZ-ring assembly and positioning in Arabidopsis through interaction with FtsZ2. Plant Cell 25(5), 1787-1802 (2013). doi:10.1105/ tpc.113.111047 
16. Glynn, J.M., Yang, Y., Vitha, S., Schmitz, A.J., Hemmes, M., Miyagishima, S.Y., Osteryoung, K.W.: PARC6, a novel chloroplast division factor, influences FtsZ assembly and is required for recruitment of PDV1 during chloroplast division in Arabidopsis. Plant J. 59(5), 700-711 (2009)

17. Olson, B.J., Wang, Q., Osteryoung, K.W.: GTP-dependent heteropolymer formation and bundling of chloroplast FtsZ1 and FtsZ2. J. Biol. Chem. 285(27), 20634-20643 (2010). doi:10.1074/jbc.M110.122614

18. Smith, A.G., Johnson, C.B., Vitha, S., Holzenburg, A.: Plant FtsZ1 and FtsZ2 expressed in a eukaryotic host: GTPase activity and self-assembly. FEBS Lett. 584(1), 166-172 (2010)

19. TerBush, A.D., Osteryoung, K.W.: Distinct functions of chloroplast FtsZ1 and FtsZ2 in Z-ring structure and remodeling. J. Cell Biol. 199(4), 623-637 (2012). doi:10.1083/jcb.201205114

20. Johnson, C.B., Shaik, R., Abdallah, R., Vitha, S., Holzenburg, A.: FtsZ1/FtsZ2 turnover in chloroplasts and the role of ARC3. Microsc. Microanal. 1-11 (2015). doi:10.1017/S1431927615000082

21. Li, Z., Trimble, M.J., Brun, Y.V., Jensen, G.J.: The structure of FtsZ filaments in vivo suggests a force-generating role in cell division. EMBO J. 26(22), 4694-4708 (2007)

22. Szwedziak, P., Wang, Q., Bharat, T.A.M., Tsim, M., Löwe, J.: Architecture of the ring formed by the tubulin homologue Fts $Z$ in bacterial cell division. Elife 3, e04601 (2014). doi:10.7554/eLife.04601

23. Li, Y., Hsin, J., Zhao, L., Cheng, Y., Shang, W., Huang, K.C., Wang, H.W., Ye, S.: FtsZ protofilaments use a hinge-opening mechanism for constrictive force generation. Science 341(6144), 392-395 (2013). doi:10.1126/ science. 1239248

24. Osawa, M., Erickson, H.P.: Liposome division by a simple bacterial division machinery. Proc. Natl. Acad. Sci. USA. 110(27), 11000-11004 (2013). doi:10.1073/pnas.1222254110

25. Loose, M., Mitchison, T.J.: The bacterial cell division proteins FtsA and FtsZ self-organize into dynamic cytoskeletal patterns. Nat. Cell Biol. 16, 38-46 (2014). doi:10.1038/ncb2885

26. Arumugam, S., Petrasek, Z., Schwille, P.: MinCDE exploits the dynamic nature of FtsZ filaments for its spatial regulation. Proc. Natl. Acad. Sci. USA. 111(13), E1192-E1200 (2014). doi:10.1073/pnas.1317764111

27. Smith, A.G., Johnson, C.B., Vitha, S., Holzenburg, A.: Oligomerization of plant FtsZ1 and FtsZ2 plastid division proteins. Arch. Biochem. Biophys. 513(2), 94-101 (2011). doi:10.1016/j.abb.2011.07.001

28. Gao, H., Kadirjan-Kalbach, D., Froehlich, J.E., Osteryoung, K.W.: ARC5, a cytosolic dynamin-like protein from plants, is part of the chloroplast division machinery. Proc. Natl. Acad. Sci. USA. 100(7), 4328-4333 (2003)

29. Miyagishima, S.Y., Froehlich, J.E., Osteryoung, K.W.: PDV1 and PDV2 mediate recruitment of the dynamin-related protein ARC5 to the plastid division site. Plant Cell 18(10), 2517-2530 (2006)

30. Fujiwara, M.T., Sekine, K., Yamamoto, Y.Y., Abe, T., Sato, N., Itoh, R.D.: Live imaging of chloroplast FtsZ1 filaments, rings, spirals, and motile dot structures in the AtMinE1 mutant and overexpressor of Arabidopsis thaliana. Plant Cell Physiol. 50(6), 1116-1126 (2009). doi:10.1093/pcp/pcp063

31. Johnson, C.B., Tang, L.K., Smith, A.G., Ravichandran, A., Luo, Z., Vitha, S., Holzenburg, A.: Single particle tracking analysis of the chloroplast division protein FtsZ anchoring to the inner envelope membrane. Microsc. Microanal. 19(3), 507-512 (2013). doi:10.1017/S143192761300038X

32. Clough, S.J., Bent, A.F.: Floral dip: a simplified method for Agrobacteriummediated transformation of Arabidopsis thaliana. Plant J. 16, 735-743 (1998)

33. Littlejohn, G.R., Gouveia, J.D., Edner, C., Smirnoff, N., Love, J.: Perfluorodecalin enhances in vivo confocal microscopy resolution of Arabidopsis thaliana mesophyll. N. Phytol. 186(4), 1018-1025 (2010)

34. Ferris, A.M., Giberson, R.T., Sanders, M.A., Day, J.R.: Advanced laboratory techniques for sample processing and immunolabeling using microwave radiation. J. Neurosci. Meth. 182(2), 157-164 (2009)

35. Stokes, K.D., McAndrew, R.S., Figueroa, R., Vitha, S., Osteryoung, K.W.: Chloroplast division and morphology are differentially affected by overexpression of Fts $Z 1$ and FtsZ2 genes in Arabidopsis. Plant Physiol. 124(4), 1668-1677 (2000)
36. McDonald, K.L., Webb, R.I.: Freeze substitution in 3 hours or less. J. Microsc. 243(3), 227-233 (2011). doi:10.1111/j.1365-2818.2011.03526x

37. Ress, D.B., Harlow, M.L., Marshall, R.M., McMahan, U.J.: Methods for generating high-resolution structural models from electron microscope tomography data. Structure 12(10), 1763-1774 (2004). doi:10.1016/j. str.2004.07.022

38. McMahan, U.J., Marshall, R., Szule, J., Jung, J.H.: Software for electron tomography. http://em3d.stanford.edu/ (2011). 2011

39. Glynn, J.M., Miyagishima, S., Yoder, D.W., Osteryoung, K.W., Vitha, S.: Chloroplast division. Traffic $\mathbf{8}(5), 451-461$ (2007)

40. Gustafsson, M.G.L., Shao, L., Carlton, P.M., Wang, C.J.R., Golubovskaya, I.N., Cande, W. Z.D., Agard, A., Sedat, J.W.: Three-dimensional resolution doubling in widefield fluorescence microscopy by structured illumination. Biophys. J. (2008). doi:10.1529/biophysj.107.120345

41. Srinivasan, R., Mishra, M., Wu, L., Yin, Z., Balasubramanian, M.K.: The bacterial cell division protein FtsZ assembles into cytoplasmic rings in fission yeast. Genes Dev. 22(13), 1741-1746 (2008). doi:10.1101/gad.1660908

42. Yoshida, Y., Kuroiwa, H., Misumi, O., Nishida, K., Yagisawa, F., Fujiwara, T., Nanamiya, H., Kawamura, F., Kuroiwa, T.: Isolated chloroplast division machinery can actively constrict after stretching. Science $\mathbf{3 1 3}(5792)$ 1435-1438 (2006). doi:10.1126/science.1129689

43. Gilkey, J.C., Staehelin, L.A.: Advances in ultra-rapid freezing for the preservation of cellular ultrastructure. J. Electron Microsc. Techn. 3(2), 177-210 (1986). doi:10.1002/jemt.1060030206

44. Kellenberger, E.: The response of biological macromolecules and supramolecular structures to the physics of specimen cryopreparation. In: Steinbrecht, R., Zierold, K. (eds.) Cryotechniques in Biological Electron Microscopy, pp. 35-63. Springer, Berlin (1987)

45. Kukulski, W., Schorb, M., Welsch, S., Picco, A., Kaksonen, M., Briggs, J.A.G.: Correlated fluorescence and 3D electron microscopy with high sensitivity and spatial precision. J. Cell Biol. 192(1), 111-119 (2011). doi:10.1083/ jcb.201009037

46. Watanabe, S., Punge, A., Hollopeter, G., Willig, K.I., Hobson, R.J., Davis, M.W., Hell, S.W., Jorgensen, E.M.: Protein localization in electron micrographs using fluorescence nanoscopy. Nat. Methods 8(1), 80-84 (2011). doi:10.1038/nmeth.1537

47. Bell, K., Mitchell, S., Paultre, D., Posch, M., Oparka, K.: Correlative imaging of fluorescent proteins in resin-embedded plant material. Plant Physiol. 161(4), 1595-1603 (2013). doi:10.1104/pp.112.212365

48. Xiong, H., Zhou, Z., Zhu, M., Lv, X., Li, A., Li, S., Li, L., Yang, T., Wang, S., Yang, Z., Xu, T., Luo, Q., Gong, H., Zeng, S.: Chemical reactivation of quenched fluorescent protein molecules enables resin-embedded fluorescence microimaging. Nat. Commun. 5 (2014). doi:10.1038/ncomms4992

49. Erickson, H.P., Taylor, D.W., Taylor, K.A., Bramhill, D.: Bacterial cell division protein FtsZ assembles into protofilament sheets and minirings, structural homologs of tubulin polymers. Proc. Natl. Acad. Sci. USA. 93(1), 519-523 (1996)

50. Lu, C.L., Reedy, M., Erickson, H.P.: Straight and curved conformations of FtsZ are regulated by GTP hydrolysis. J. Bacteriol. 182(1), 164-170 (2000)

51. Popp, D., Iwasa, M., Narita, A., Erickson, H.P., Maeda, Y.: FtsZ condensates: an in vitro electron microscopy study. Biopolymers 91(5), 340-350 (2009)

52. Mingorance, J., Rivas, G., Velez, M., Gomez-Puertas, P., Vicente, M.: Strong FtsZ is with the force: mechanisms to constrict bacteria. Trends Microbiol. 18(8), 348-356 (2010). doi:10.1016/j.tim.2010.06.001

53. Crowther, R.A., DeRosier, D.J., Klug, A.: The reconstruction of a threedimensional structure from projections and its application to electron microscopy. Proc. Roy. Soc. Lond. A. 317(1530), 319-340 (1970). doi:10.1098/rspa.1970.0119 